\title{
EFFECTS OF BALLISTIC TRANSPORT IN WIRES OF $n$-PbTe
}

\author{
G. Grabecki, J. Wróbel, T. Dietl \\ Institute of Physics, Polish Academy of Sciences \\ Al. Lotników 32/46, 02-668 Warszawa, Poland \\ E. Papis, E. Kamińska, A. Piotrowska \\ Institute of Electron Technology, Al. Lotników 32/46, 02-668 Warszawa, Poland \\ Y. Ueta, G. Sprinholtz and G. Bauer \\ Institut für Halbleiterphysik, Johannes Kepler Universität Linz, 4040 Linz, Austria
}

We present results of magnetotransport studies on quantum wires of submicron $\mathrm{PbTe}$ epilayers, fabricated by means of electron beam lithography and dry etching. When the wire width is reduced down to $1 \mu \mathrm{m}$, the transition from diffusive to ballistic regime is observed. Effects associated with collimation and boundary scattering are found in the Hall, longitudinal, and van der Pauw magnetoresistance for wires and junctions in the shape of a cross.

PACS numbers: 73.23.Ad, 73.61.-r, 73.50.Dn

Electron transport in metal and semiconductor structures, in which the geometrical length $L$ becomes smaller than the mean free path of the carriers, $l_{\mathrm{e}}$, is known as the ballistic regime. In this regime carriers scarcely suffer scatterings and can transmit straight forward along the accelerating electric field until they bump against the wall. Since the sample boundaries affect directly the motion of the carriers, the conductance is sensitive to geometrical shape of the device. Up to now, the ballistic regime has been achieved only in very pure metals and nanostructures made of high mobility two-dimensional electron gas at III-V semiconductor heterostructures [1]. In the present work, signatures of ballistic transport are put into the evidence in nanostructures of a lead chalcogenide material system. These materials, such as $\mathrm{PbTe}$, seem to be excellent candidates for search of ballistic effects because of an extremely large dielectric constant $(\epsilon \approx 1000)$ and low effective mass $\left(m^{*}=0.021 m_{0}\right)$, which make $l_{\mathrm{e}}$ to be as long as several micrometers [2].

The starting material consists of $n$-PbTe:Bi [111] films with the thickness of $0.2 \mu \mathrm{m}$, grown by $\mathrm{MBE}$ onto $\mathrm{BaF}_{2}$ substrate and $0.2 \mu \mathrm{m} \mathrm{Pb}_{0.94} \mathrm{Eu}_{0.06} \mathrm{Te}$ buffer layer. The structure completes an $0.05 \mu \mathrm{m} \mathrm{Pb}_{0.94} \mathrm{Eu}_{0.06} \mathrm{Te}$ cap layer. The buffer 
layer serves to accommodate misfit dislocations and to reduce strain resulting from the mismatch of the thermal expansion coefficients between the substrate and the films [3]. The wires in the form of six-terminal Hall bridges, with lateral width $W$ ranging from $100 \mu \mathrm{m}$ down to $1 \mu \mathrm{m}$, were fabricated by electron beam lithography followed by Ar ion milling. Electron mobility in the studied wires is typically $10 \mathrm{~m}^{2} /(\mathrm{V} \mathrm{s})$ which corresponds to $l_{\mathrm{e}}$ of about $1.2 \mu \mathrm{m}$.
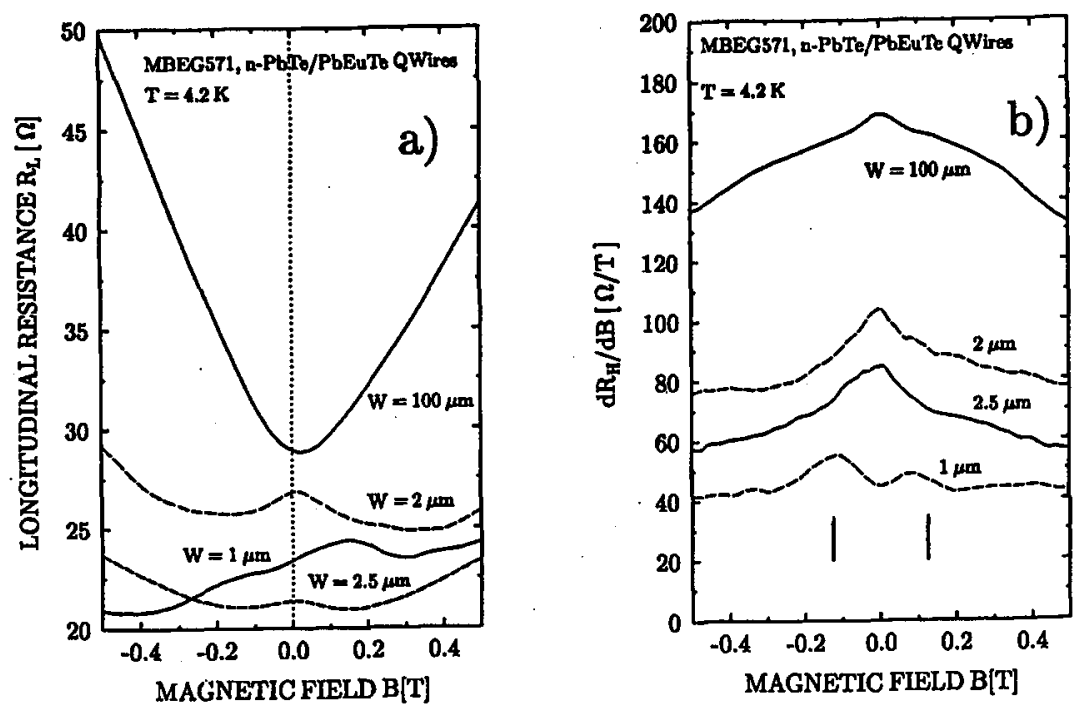

Fig. 1. (a) The magnetoresistance of $\mathrm{PbTe} / \mathrm{PbEuTe}$ wires of differing widths at $4.2 \mathrm{~K}$. (b) First derivative of the Hall resistance for the same set of wires. Arrows indicate the magnetic field values corresponding to $l_{\text {cycl }}=1 \mu \mathrm{m}$.

Longitudinal magnetoresistances for $\mathrm{PbTe}$ wires of diferent widths are shown in Fig. 1a. In the macroscopic wire $(W=100 \mu \mathrm{m})$, only a positive magnetoresistance is observed. Its presence indicates multi-channel transport, which may result from the multi-valley band structure and/or strain induced edge transport [3]. However, in the narrower wires, additional negative magnetoresistance peak appears near zero-magnetic field. This is interpreted in terms of the magnetic field-induced suppression of the diffusive part of the boundary scattering [4]. The magnitude of the magnetoresistance peak allows us to estimate $p$ - the mutual ratio between the specular and diffusive contributions to the boundary scattering. For example, in the case of $2 \mu \mathrm{m}$ wire, the resistance change in the magnetic field is about $10 \%$ of the total zero-field resistance. According to the model of Beenakker et al. [4] we evaluate $p=0.80 \pm 0.1$. A similar value was observed in wires of $\mathrm{AlGaAs} / \mathrm{GaAs}$ patterned by reactive ion etching [5].

In the narrowest wire $(W=1 \mu \mathrm{m})$ a splitting of the magnetoresistance peak becomes visible. This may be an indication of a magneto size effect, appearing if the cyclotron radius, 


$$
l_{\mathrm{cycl}}=\frac{\hbar k_{\mathrm{F}}}{e B}
$$

becomes comparable with the wire width. In our case $l_{\text {cycl }}=W$ at $B=0.13 \mathrm{~T}$, which is consistent with the positions of the two broad maxima in Fig. 1a.

The first derivative of the Hall resistance (the Hall slope) for the same set of wires is shown in Fig. 1b. A strong decrease in a mean Hall constant with reducing $W$ indicates an increase in electron concentration in the narrow wires. This is probably due to an enhancement of stress in small structures [6]. Similarly to the case of longitudinal resistance, the Hall slope for the $1 \mu \mathrm{m} \mathrm{PbTe}$ wire exhibits two distinct peaks at about $B \approx \pm 0.13 \mathrm{~T}$, a value in agreement with the condition $l_{\text {cycl }} \approx W$. While the theoretical model for the ballistic Hall structures [7] predicts such behavior, its character depends strongly on details of the actual wire geometry and boundaries. In our case, a possible nonuniform strain and effects of the band anisotropy complicates theoretical analysis even further.

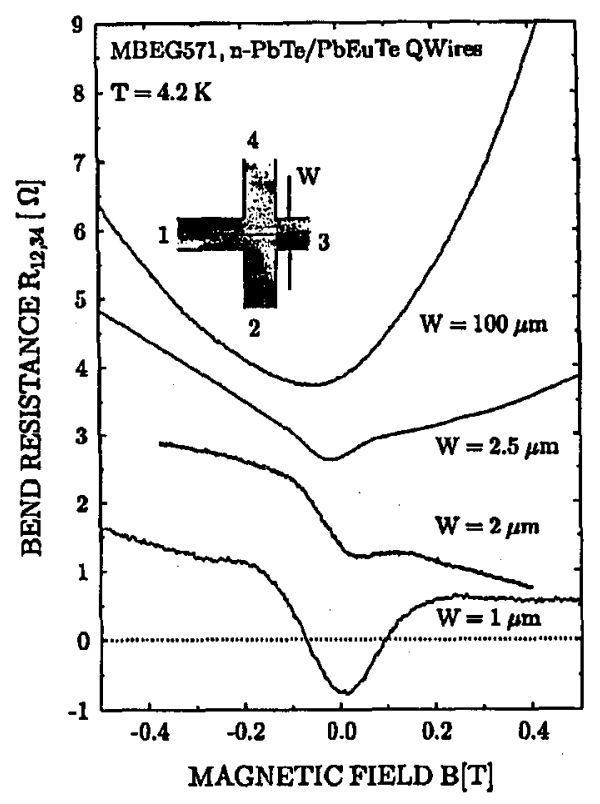

Fig. 2. Bend resistance of $\mathrm{PbTe} / \mathrm{PbEuTe}$ wires of differing widths at $4.2 \mathrm{~K}$. Inset shows the contact probe geometry.

Figure 2 shows the field dependence of the so-called bend resistance. The corresponding contact geometry is shown in the inset. The current flows through two adjacent contacts 1 and 2 , and the voltage is measured on the remaining pair of contacts, 3 and 4 . In the macroscopic wires, such an arrangement corresponds to the well-known van der Pauw geometry. As $W$ is reduced, a resistance minimum develops in the vicinity of the zero-magnetic field. For the $1 \mu \mathrm{m}$ wire, the resistance is seen to become negative. This is a clear manifestation of the ballistic motion of the electrons through the node. Indeed, the electrons injected by contact 1 travel 
directly to contact 3 (not 2), so that the sign of the measured potential $V_{3}-V_{4}$ is reversed. However, under the presence of the magnetic field for which $l_{\text {cycl }} \leq W$, the electron trajectories are bent by the Lorentz force, and the positive value of resistivity is restored [8].

It should be pointed out that all the above effects are most pronounced for the $1 \mu \mathrm{m}$ wire, in which the condition $l_{\mathrm{e}}>W$ is well fulfilled, so that the true ballistic regime is reached. At the same time, a change of temperature in the range from $4.2 \mathrm{~K}$ down to $50 \mathrm{mK}$ is found to not affect the observed phenomena. This is because the ballistic effects are controlled by $l_{e}$ which ceases to vary with temperature in this range. This is in stark contrast to quantum phenomena in the diffusive regime (such as weak localization or universal conductance fluctuations), whose magnitude is determined by the inelastic scattering length that increases strongly with lowering temperature.

This work was partially supported by the Committee for Scientific Research through grant 2PO3B06411.

\section{References}

[1] C.W.J. Beenakker, H. van Houten, Solid State Physics, Vol. 44, Academic, Boston 1991, p. 1.

[2] J.I. Ravich, B.A. Efimova, I.A. Smirnov, Semiconducting Lead Chalcogenides, Plenum, New York 1970.

[3] G. Grabecki, J. Wróbel, T. Dietl, M. Sawicki, J. Domagała, T. Skośkiewicz, E. Papis, E. Kaminiska, A. Piotrowska, M. Leszczyński, Y. Ueta, G. Sprinholtz, G. Bauer, Superlatt. Microstruct. 22, 51 (1997); Acta Phys. Pol. A 90, 797 (1996).

[4] C.W.J. Beenakker, H. van Houten, Solid State Physics, Vol. 44, Academic, Boston 1991, Ch. II.5, p. 26.

[5] T.J. Thornton, M.L. Roukes, A. Scherer, B.P. Van de Gaag, Phys. Rev. Lett. 63, 2128 (1989).

[6] A. Atkinson, K. Pinardi, S.C. Jain, Semicond. Sci. Technol. 11, 1271 (1996).

[7] C.J.B. Ford, S. Washburn, M. Büttiker, C.M. Knoedler, J.M. Hong, Surf. Sci. 229, 298 (1990).

[8] C.W.J. Beenakker, H. van Houten, Solid State Physics, Vol. 44, Academic, Boston 1991, Ch. III.16, p. 146. 